\title{
Paracrine signaling in stem cell renewal and in neoplastic tumor growth
}

\author{
ZHAO Chen \\ Department of Developmental Biology, Stanford University, CA 94305, USA \\ Received February 15, 2014; accepted April 2, 2014; published online May 20, 2014
}

\begin{abstract}
Paracrine pathway activities are being increasingly recognized as instrumental regulatory mechanisms of epithelial-stromal interactions that play important roles in physiological and pathological self-renewal of stem cells and in the initiation and maintenance of neoplastic tumor development. Stromal-specific Hedgehog (Hh) responses and epithelial-associated Wnt pathway activities have been recently appreciated as important factors in stem cell self-renewal and carcinogenesis. Furthermore, Hh and Wnt pathways frequently crosstalk with each other to regulate the growth of epithelial cells in a context-dependent manner. Because small molecule modulators of $\mathrm{Hh}$ and Wnt pathway activities are readily available, emerging roles of Hh-Wnt pathway crosstalk in epithelial-stromal interactions will shed light on the development of regenerative and anti-cancer medicines.
\end{abstract}

Hedgehog, Wnt, paracrine, stem cell, tumor

Citation: Zhao C. Paracrine signaling in stem cell renewal and in neoplastic tumor growth. Sci China Life Sci, 2014, 57: 571-574, doi: $10.1007 / \mathrm{s} 11427-014-4664-8$

Epithelial and stromal interactions play critical roles in embryonic development, adult tissue homeostasis [1], and injury-induced regeneration [2]. Deregulation of epithelial-stromal interactions is often associated with diseases that affect epithelial cell growth, such as in tumorigenesis [1]. Despite progress in understanding the activities and roles of epithelial-stromal interactions in various tissues and diseases, little has been known about the signaling pathways that directly regulate epithelial-stromal crosstalk. Accumulating evidence has highlighted the underappreciated roles of Wnt and Hedgehog $(\mathrm{Hh})$ signaling pathways in epithelial-stromal interactions [3]. In this review, we focus on the recent progress in understanding the roles of Wnt and $\mathrm{Hh}$ pathway activities in epithelial-stromal interactions, the crosstalk between Wnt and Hh pathways during physiological tissue homeostasis, and carcinogenesis. We also briefly summarize the recent use of $\mathrm{Hh}$ and Wnt pathway modulators as

*Corresponding author (email: chenzhao@stanford.edu) anti-cancer medicines and their potential application to target epithelial-stromal interactions in pathological conditions.

The Hh signaling pathway plays critical roles in embryogenesis and adult tissue homeostasis [4,5]. The paradigm of how Hh signaling is normally operated in various tissues is that Hh ligands secreted from epithelial cells act in a non-cell autonomous manner to elicit supportive $\mathrm{Hh}$ responses in stromal cells, which ultimately feedback to affect epithelial cell growth [6]. This circuit involves feedback interactions between Hh-producing epithelial cells and Hh-responsive stromal cells. Because Hh signaling is one of the instrumental cues that regulate both embryonic development and homeostasis of multiple adult tissues, deregulation of Hh signaling is often associated with developmental syndromes and carcinogenesis [7,8]. During embryonic development, loss-of-Hh pathway activity due to Sonic Hedgehog (SHH) or GLI2 gene mutations leads to holoprosencephaly and multiple pituitary hormone deficiency, 
respectively. On the other hand, gain-of-Hh pathway activity resulting from germline mutations of Smoothened (SMO) and Patched (PTCH1) can cause basal cell carcinoma (BCC) and medulloblastoma. Although $\mathrm{Hh}$ signaling was initially reported to act cell-autonomously in the initiation and growth of neural and epithelial tumors such as medulloblastoma and BCC $[9,10]$, accumulating evidence suggests that $\mathrm{Hh}$ signaling could also function in a paracrine manner in other solid tumors to regulate their growth [7], in which Hh-responsive cells are restricted to tumor-associated stromal cells and tumor epithelial cells secrete Hh ligands. Furthermore, in response to $\mathrm{Hh}$ stimulation, tumor-associated stromal cells secrete soluble proteins that induce either proliferation or differentiation of epithelial tumors. The net effect of proliferation and differentiation signals induced by Hh-responsive cells in the initiation and growth of epithelial tumors is currently under extensive investigation in various mouse models of human cancers. Consistent with the requirement for Hh signaling in early development, roles of Hh signaling in the maintenance of adult stem cells/progenitor cells have been gradually recognized in recent years. Intriguingly, the Hh response acts on stem/progenitor cells directly, such as neural precursors [11] and hematopoietic stem cells (HSCs) [12], although exact mechanisms of Hh signaling in HSC maintenance and regeneration are still unclear. Hh signaling can alternatively act in a paracrine manner to regulate stem cell homeostasis in a specific tissue. A recent study [3] employed mouse genetic approaches to reveal extensive crosstalk between Shh-expressing epithelial cells with enriched stem cell units and Hh-responsive stromal cells in homeostasis and a bacterial injury-induced model of pathological regeneration of the bladder urothelium. Despite progress in identifying either Hh-secreting or Hh-responding cells among the above-mentioned adult stem cells, the absolute proportions of adult stem cells that secrete $\mathrm{Hh}$ ligands or respond to $\mathrm{Hh}$ stimulation are largely unknown. Clear understanding of this aspect by co-staining or fluorescence-activated cell sorting (FACS) with stem cell markers would help to clarify the specific expression of $\mathrm{Hh}$ pathway components in adult stem cells.

As a closely related signaling pathway that also plays critical roles in embryogenesis and adult tissue homeostasis, the Wnt signaling pathway has well-characterized activities in tissue-specific stem cells [13] and the development of various epidermal-derived tumors [14]. Intriguingly, compared with the predominant model in which Hh-responsive stromal cells affect the growth of epithelial stem cells and epithelial tumor cells that secrete $\mathrm{Hh}$ ligands, the Wnt pathway response is frequently restricted in stem cells and epithelial tumor cells. In contrast to the epithelial-specific expression pattern of Wnt responsive cells, Wnt ligands can be expressed in either stromal cells or Wnt-responsive epithelial cells. In the former, the Wnt pathway acts via a similar paracrine mechanism as the above-described Hh pathway, except that Wnt pathway responses occur in epithelial cells and Wnt ligands are secreted from stromal cells. However, in the latter case, Wnt pathway activity acts cell-autonomously to regulate epithelial stem cell and cancer cell growth. Notably, compared with the three Hh ligands (Shh, Ihh, and Dhh) in mammalian cells, the Wnt ligand family includes $19 \mathrm{Wnt}$ proteins that execute their biological activities in tissue- and context-dependent manners [15]. A clear example of Wnt pathway activity in stem cell and tumor growth is its well-documented roles in the maintenance of mammary stem cells and carcinogenesis of mammary tumors. Recent studies [16,17] have employed FACS and lineage-labeling technologies to identify Wntresponsive cells as a subset of mammary epithelial cells with enriched mammary regenerative units (MRU). Wnt signaling activity potentiates the MRU of mammary stem cells as evidenced by the activity of exogenous Wnt protein to clonally expand mammary stem cells in vitro and to maintain their ability to generate functional mammary glands in in vivo transplantation assays. Because a proper regulated level of Wnt signaling is critical to maintain the MRU of mammary epithelial cells, deregulated Wnt pathway activity in mammary epithelial cells is frequently associated with the development and growth of human breast cancer [18]. Importantly, genetic up-regulation of Wnt pathway activity in mouse mammary epithelial cells via mammary epithelial-specific drivers such as mouse mammary tumor virus (MMTV) leads to the formation of mammary adenocarcinoma [19] in a mouse model (MMTV-Wnt) of breast cancer. This finding highlights the critical cell-autonomous role of Wnt in breast cancer development.

Although the above-mentioned epithelial Wnt and stromal $\mathrm{Hh}$ responses contribute to epithelial-stromal interactions in different cellular compartments, their activities are similarly required during embryonic development and adult tissue homeostasis. Thus, it is important to determine in a given tissue where epithelial Wnt and stromal $\mathrm{Hh}$ pathway activities are both active whether these pathway activities can reciprocally crosstalk with each other. Indeed, Wnt and Hh pathway activities negatively regulate each other in neural tube development [20]. Wnt is secreted from the ventral spinal cord and specifies ventral cell fates via its antagonistic activity that represses dorsally restricted $\mathrm{Hh}$ pathway activity. Conversely, a Hh response is initiated in the floor plate of the neural tube and determines dorsal cell fates via its antagonistic activity that represses Wnt activity. Intriguingly, a recent study [3] describes unexpected positive crosstalk of $\mathrm{Hh}$ and Wnt pathways in the adult urothelium. Shh is secreted from basal cells of the bladder urothelium, which up-regulates Wnt and Fgf ligand secretion in Shh-responsive stromal cells, while secreted Wnt ligands reciprocally promote the growth of urothelial epithelium (Figure 1). Importantly, this circuit of Wnt-Hh crosstalk is required for regenerative activity of the bladder urothelium in response to bacterial injury because genetic or pharmacological interventions of this Wnt-Hh crosstalk delay such 
regenerative proliferation. It would be interesting to examine the significance of this type of positive interaction between Wnt and Hh signaling in the initiation and maintenance of bladder tumors. Furthermore, it would be useful to determine whether this type of Wnt-Hh crosstalk occurs in other epithelial-stromal interactions that similarly involve epithelial Wnt responses and stromal $\mathrm{Hh}$ responses (Figure 1).

Because paracrine activities of $\mathrm{Hh}$ and $\mathrm{Wnt}$ are required for stem cell maintenance and epithelial tumor growth, pharmacological activation or inhibition of these pathways would have therapeutic value for diseases driven by deregulated activities of Wnt or Hh pathways. Both $\mathrm{Hh}$ and Wnt pathway activities are initiated by $\mathrm{G}$ protein-coupled receptor (GPCR)-like membrane receptors/co-receptors that can be targeted easily by drugs. Therefore, extensive efforts have been made to identify $\mathrm{Hh}$ and Wnt pathway-specific antagonists in small molecule libraries. As a result of high throughput analyses, non-toxic anti-Hh drug candidates are currently being tested in over 40 clinical trials sponsored by six major pharmaceutical companies for the treatment of brain, skin, and visceral cancers including medulloblastoma, BCC, pancreatic adenocarcinoma, and lung cancer. The most advanced anti-Hh drug candidate, Vismodegib, from Genentech was approved by the Food and Drug Administration on 2012 [21]. Compared with Hh pathway antagonists that mainly target membrane GPCR-like receptor in the Hh pathway, approaches to manipulate the Wnt pathway range from targeting critical regulators of Wnt secretion to essential mediators of the Wnt response [22]. The activities of these Wnt pathway modulators in various diseases are currently under investigation by major pharmaceutical companies. Considering the diversity of Wnt ligands and models in which the Wnt pathway can be operated in either paracrine or autocrine manners, the efficacy of these Wnt pathway modulators may have to be evaluated in a context-dependent manner.

Accumulating data have revealed that tumor epithelial cells are consistently exposed to and require growth signals from tumor-associated stromal cells. Such findings undoubtedly suggest an alternative strategy to inhibit tumor growth by interruption of the epithelial-stromal interactions required for tumor growth. Identification of $\mathrm{Hh}$ and Wnt pathway crosstalk as an essential regulatory mechanism in epithelial-stromal interactions will shed light on the development of regenerative and anti-cancer medicines. Exciting
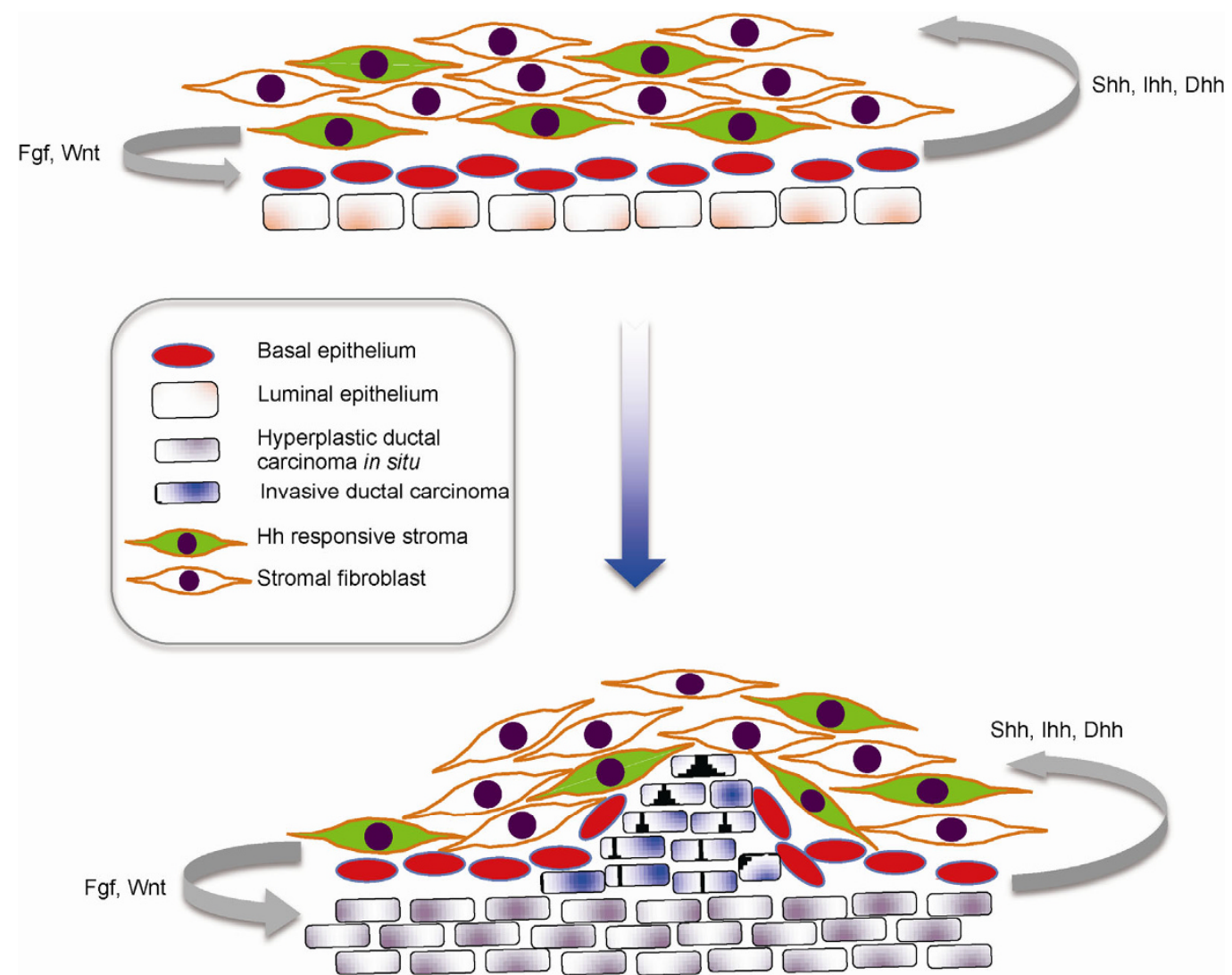

Figure 1 According to a recent study [3], Shh is secreted from basal cells of the bladder urothelium and Hh-responsive cells in the surrounding stroma secrete soluble factors, such as Fgf and Wnt family proteins, which can feedback to promote bladder urothelial proliferation. This crosstalk acts to protect the bladder from bacterial injury-induced degeneration. However, the exact role remains to be determined for this Hh-Wnt crosstalk circuit in the initiation and maintenance of bladder tumors. 
time is clearly ahead as Hh-Wnt crosstalk is implicated in regulating physiological and pathological epithelial-stromal interactions.

1 Polyak K, Kalluri R. The role of the microenvironment in mammary gland development and cancer. Cold Spring Harb Perspect Biol, 2010, 2: a003244

2 Gabison EE, Huet E, Baudouin C, Menashi S. Direct epithelialstromal interaction in corneal wound healing: role of EMMPRIN/ CD147 in MMPs induction and beyond. Prog Retin Eye Res, 2009, 28: 19-33

3 Shin K, Lee J, Guo N, Kim J, Lim A, Qu L, Mysorekar IU, Beachy PA. Hedgehog/Wnt feedback supports regenerative proliferation of epithelial stem cells in bladder. Nature, 2011, 472: 110-114

4 Varjosalo M, Taipale J. Hedgehog: functions and mechanisms. Genes Dev, 2008, 22: 2454-2472

5 van den Brink GR. Hedgehog signaling in development and homeostasis of the gastrointestinal tract. Physiol Rev, 2007, 87: 1343-1375

6 Shaw A, Bushman W. Hedgehog signaling in the prostate. J Urol, 2007, 177: 832-838

7 Theunissen JW, de Sauvage FJ. Paracrine hedgehog signaling in cancer. Cancer Res, 2009, 69: 6007-6010

8 Gorlin RJ. Nevoid basal cell carcinoma (gorlin) syndrome. Genet Med, 2004, 6: 530-539

9 Goodrich LV, Milenkovic L, Higgins KM, Scott MP. Altered neural cell fates and medulloblastoma in mouse patched mutants. Science, 1997, 277: 1109-1113

10 Xie J, Murone M, Luoh SM, Ryan A, Gu Q, Zhang C, Bonifas JM, Lam CW, Hynes M, Goddard A, Rosenthal A, Epstein EH Jr, de Sauvage FJ. Activating smoothened mutations in sporadic basal-cell carcinoma. Nature, 1998, 391: 90-92

11 Bambakidis NC, Wang RZ, Franic L, Miller RH. Sonic hedgehog- induced neural precursor proliferation after adult rodent spinal cord injury. J Neurosurg, 2003, 99: 70-75

12 Zhao C, Chen A, Jamieson CH, Fereshteh M, Abrahamsson A, Blum J, Kwon HY, Kim J, Chute JP, Rizzieri D, Munchhof M, VanArsdale $\mathrm{T}$, Beachy PA, Reya T. Hedgehog signalling is essential for maintenance of cancer stem cells in myeloid leukaemia. Nature, 2009, 458: 776-779

13 Nusse R, Fuerer C, Ching W, Harnish K, Logan C, Zeng A, ten Berge D, Kalani Y. Wnt signaling and stem cell control. Cold Spring Harb Symp Quant Biol, 2008, 73: 59-66

14 Polakis P. Wnt signaling in cancer. Cold Spring Harb Perspect Biol, 2012, 4: a008052

15 Willert K, Nusse R. Wnt proteins. Cold Spring Harb Perspect Biol, 2012, 4: a007864

16 Zeng YA, Nusse R. Wnt proteins are self-renewal factors for mammary stem cells and promote their long-term expansion in culture. Cell Stem Cell, 2010, 6: 568-577

17 van Amerongen R, Bowman AN, Nusse R. Developmental stage and time dictate the fate of Wnt/beta-catenin-responsive stem cells in the mammary gland. Cell Stem Cell, 2012, 11: 387-400

18 Prosperi JR, Goss KH. A Wnt-ow of opportunity: targeting the Wnt/beta-catenin pathway in breast cancer. Curr Drug Targets, 2010, 11: 1074-1088

19 Shackleford GM, MacArthur CA, Kwan HC, Varmus HE. Mouse mammary tumor virus infection accelerates mammary carcinogenesis in Wnt-1 transgenic mice by insertional activation of int-2/Fgf-3 and hst/Fgf-4. Proc Natl Acad Sci USA, 1993, 90: 740-744

$20 \mathrm{Yu}$ W, McDonnell K, Taketo MM, Bai CB. Wnt signaling determines ventral spinal cord cell fates in a time-dependent manner. Development, 2008, 135: 3687-3696

21 Dlugosz A, Agrawal S, Kirkpatrick P. Vismodegib. Nat Rev Drug Discov, 2012, 11: 437-438

22 Chen B, Dodge ME, Tang W, Lu J, Ma Z, Fan CW, Wei S, Hao W, Kilgore J, Williams NS, Roth MG, Amatruda JF, Chen C, Lum L. Small molecule-mediated disruption of Wnt-dependent signaling in tissue regeneration and cancer. Nat Chem Biol, 2009, 5: 100-107

Open Access This article is distributed under the terms of the Creative Commons Attribution License which permits any use, distribution, and reproduction in any medium, provided the original author(s) and source are credited. 